\title{
The break-up of a liquid cylinder in a centrifugal field
}

\author{
H.N.W. Lekkerkerker*, E.H.A. de Hoog \\ Van 't Hoff laboratory, Utrecht University, Debye Institute, Padualaan 8, $3584 \mathrm{CH}$ Utrecht,
} The Netherlands

\begin{abstract}
A simple model for the effect of a centrifugal field on the break-up of a long liquid cylinder is developed. The results are in qualitative agreement with recent observations. (C) 2001 Elsevier Science B.V. All rights reserved.
\end{abstract}

\section{Introduction}

Dick Bedeaux has made significant contributions to the theory and understanding of interfaces in liquids and to the low-Reynolds number hydrodynamics of dispersions. He also has a vivid interest in the teaching of the fundamental aspects of these subjects. We felt it appropriate therefore to contribute to this volume, dedicated to him on the occasion of his 60th birthday, a paper which deals with both aspects and which is largely of a didactic nature.

Recently we studied the interfacial tension between coexisting phases in a phaseseparated colloid-polymer mixture [1,2]. A crude estimate of the interfacial tension $\gamma$ is given by the simple scaling relation $[3,4]$.

$$
\gamma \sim \frac{k_{\mathrm{B}} T}{\xi^{2}}
$$

in which $k_{\mathrm{B}}$ is the Boltzmann constant, $T$ is the temperature and $\xi$ is a length scale related to the interfacial thickness. Far from the critical point the interfacial thickness is about the size of the colloid [2] and we expect interfacial tensions of the order of $1-10 \mu \mathrm{N} / \mathrm{m}$.

\footnotetext{
* Corresponding author. Tel.: 030-2532547/2391; fax: 030-2533870.

E-mail address: h.n.w.lekkerkerker@chem.uu.nl (H.N.W. Lekkerkerker).
} 
A convenient and accurate method to determine ultralow interfacial tensions between two fluid phases is the spinning drop method [5,6]. The spinning drop method is based on the fact that the elongation of a liquid droplet of a lighter fluid suspended in a heavier fluid due to centrifugal forces is balanced by the interfacial tension between the two phases. At sufficiently high centrifugal fields the droplet becomes cylindrical capped with two hemispheres. The radius of these spherical caps is equal to $2 / 3$ of the radius $R$ of the cylinder. In 1942 Vonnegut [5] showed that in the limit of cylindrical droplets the interfacial tension $\gamma$ is related to the radius $R$ of the liquid cylinder, the rotational speed $\omega$ and the density difference $\Delta \rho$ between the two phases by the relation

$$
\gamma=\frac{\omega^{2} R^{3} \Delta \rho}{4}
$$

We observed that the elongated droplets which are produced in the spinning drop apparatus break up into several smaller droplets when the rotational speed is sufficiently reduced. A careful analysis revealed that the rate of break-up and the wavelength of the instability depend strongly on the final rotational speed [9]. In this paper we present a simple model that accounts for these effects.

\section{The instability of a liquid cylinder}

As early as in 1878, Rayleigh [7] treated the instability of a long, thin, liquid cylinder and this work was extended by Tomotika [8] in 1935. Consider a long liquid cylinder with radius $R_{0}$ and viscosity $\eta_{1}$ surrounded by another liquid with viscosity $\eta_{2}$.

The breakup is driven by pressure differences in the droplet; the pressure in a neck (position A in Fig. 1) is higher than in a bulge (position B in Fig. 1) with respect to points $\mathrm{C}$ and $\mathrm{D}$. Points $\mathrm{C}$ and $\mathrm{D}$ are positions in the surrounding fluid phase (see Fig. 1). The fluid will flow from point $A$ to point $B$ with a certain velocity, which depends on the magnitude of the pressure difference, the viscosities of both fluids and the initial radius of the drop. The pressure differences are determined by the Laplace equation

$$
p=\gamma\left(\frac{1}{R_{1}}+\frac{1}{R_{2}}\right) .
$$

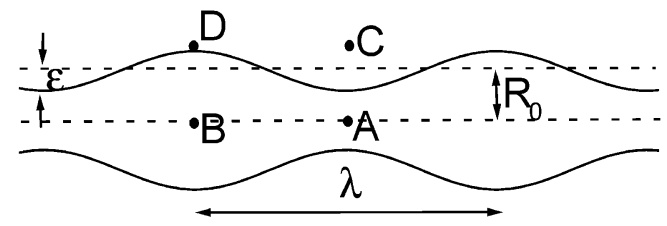

Fig. 1. Schematic representation of a breaking droplet. 
Here $R_{1}$ and $R_{2}$ are the radii of curvature of the interface. For a liquid cylinder these can be expressed as a function of the deformation.

$$
R(z)=R_{0}+\varepsilon \sin \left(\frac{2 \pi z}{\lambda}\right)
$$

where the $z$-direction is perpendicular to the axis of rotation. The pressure in point $\mathrm{A}$ with respect to point $\mathrm{C}$ is

$$
\begin{aligned}
p_{\mathrm{A}} & =p_{\mathrm{C}}+\gamma\left[\frac{1}{R_{0}-\varepsilon}+\left(-\frac{\mathrm{d}^{2} R}{\mathrm{~d} z^{2}}\right)\right] \\
& =p_{\mathrm{C}}+\frac{\gamma}{R_{0}}\left[1+\frac{\varepsilon}{R_{0}}-\varepsilon R_{0}\left(\frac{2 \pi}{\lambda}\right)^{2}\right] .
\end{aligned}
$$

Similarly, the pressure in point $\mathrm{B}$ with respect to point $\mathrm{D}$ is

$$
p_{\mathrm{B}}=p_{\mathrm{D}}+\frac{\gamma}{R_{0}}\left[1-\frac{\varepsilon}{R_{0}}+\varepsilon R_{0}\left(\frac{2 \pi}{\lambda}\right)^{2}\right] .
$$

Assuming that the pressure in points $\mathrm{C}$ and $\mathrm{D}$ is equal, the pressure difference (due to the difference in curvature of the interface) is

$$
\Delta p_{\text {Laplace }}=p_{\mathrm{A}}-p_{\mathrm{B}}=\frac{2 \gamma \varepsilon}{R_{0}^{2}}\left(1-X^{2}\right),
$$

where $X=2 \pi R_{0} / \lambda$.

A droplet will break when this pressure difference is larger than zero, which is true for $X<1$. Thus the wavelength should be larger than the circumference of the drop $\left(\lambda>2 \pi R_{0}\right)$.

The calculation of the initial growth rate of deformation (4) was performed by Tomatika [7]. Here we present a simple approximate calculation of the growth rate based on Poiseuille's formula for pipe flow. According to Poiseuille's formula the volume flux $Q$ of a liquid with viscosity $\eta_{1}$ in a pipe with radius $R_{0}$ under the influence of a pressure gradient $\Delta p / l$ is given by

$$
Q=\frac{\pi R_{0}^{4} \Delta p}{8 \eta_{1} l} .
$$

In order for the amplitude $\varepsilon$ of deformation (4) to grow by an amount $\mathrm{d} \varepsilon$ a volume of liquid

$$
\mathrm{d} V=\lambda R_{0} \mathrm{~d} \varepsilon
$$

has to flow from $\mathrm{A}$ to $\mathrm{B}$. We now put the rate of change of $\mathrm{d} V$ equal to the volume flux

$$
\frac{\mathrm{d} V}{\mathrm{~d} t}=Q
$$

Using for $Q$ Eq. (8) with $\Delta p$ given by Eq. (7) and $l=\lambda / 2$ we obtain

$$
\lambda R_{0} \frac{\mathrm{d} \varepsilon}{\mathrm{d} t}=\frac{\gamma \varepsilon\left(1-X^{2}\right) \pi R_{0}^{2}}{2 \eta_{1} \lambda} .
$$




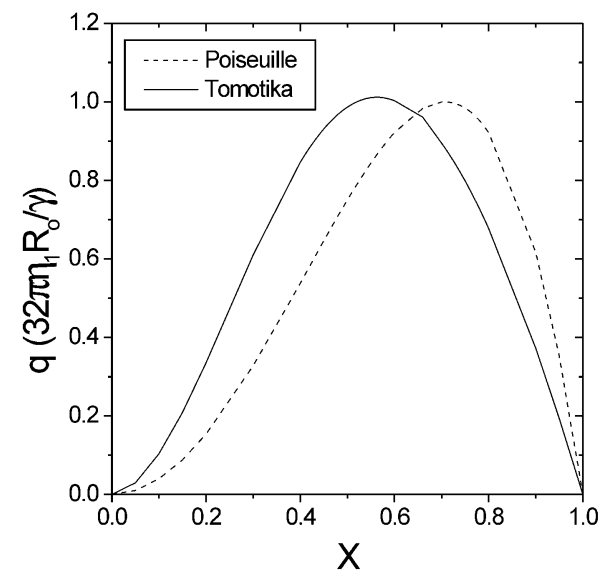

Fig. 2. Growth rate as a function of the inverse wavelength.

The solution of this equation is

$$
\varepsilon=\varepsilon(0) \mathrm{e}^{q t}
$$

with the growth rate

$$
q=\frac{\gamma X^{2}\left(1-X^{2}\right)}{8 \pi \eta_{1} R_{0}} .
$$

It is interesting to compare this result to the exact expression for the growth rate obtained by Tomotika [8]

$$
q=\frac{\gamma\left(1-X^{2}\right)}{2 R_{0} \eta_{2}} \Phi\left(\frac{\eta_{2}}{\eta_{1}}, X\right),
$$

where $\Phi$ is a function of the viscosity ratio of the outer and inner fluid and $X$.

Although quantitatively different the approximate and exact expression for $q$ qualitatively have the same behaviour. In Fig. 2 we compare them for the case $\eta_{2} / \eta_{1}=11.6$ the value that applies to our experiments [1].

Following Rayleigh we identify the observed wavelength of the instability with that of the maximum growth rate.

From Eq. (13) we obtain

$$
X_{\mathrm{m}}=\frac{1}{\sqrt{2}}
$$

and

$$
q_{\mathrm{m}}=\frac{\gamma}{32 \pi \eta_{1} R_{0}}
$$

For $\eta_{2} / \eta_{1}=11.6$ the Tomotika expression yields

$$
X_{\mathrm{m}}=0.56
$$


and

$$
q_{\mathrm{m}}=1.012 \frac{\gamma}{32 \pi \eta_{1} R_{0}} .
$$

Clearly the simple model considered here for the viscosity ratio $\eta_{2} / \eta_{1}=11.6$ gives results in good agreement with the full hydrodynamic solution of the problem.

\section{Instability of a liquid cylinder in a centrifugal field}

Now, assume that in the final stage there is a finite rotational speed causing a centrifugal field. This influences the pressure difference, which in turn effects the growth rate. The contribution of the centrifugal field to the pressure difference can be determined as (which is clear by the analysis of the pressure difference along the path $\mathrm{A}-\mathrm{C}$ compared to the path B-D in Fig. 1):

$$
\begin{aligned}
\Delta p_{\text {centr. }}= & \frac{1}{2} \omega^{2} \rho_{1}\left(R_{0}+\varepsilon\right)^{2} \\
& -\left\{\frac{1}{2} \omega^{2} \rho_{1}\left(R_{0}-\varepsilon\right)^{2}+\frac{1}{2} \omega^{2} \rho_{2}\left[\left(R_{0}+\varepsilon\right)^{2}-\left(R_{0}-\varepsilon\right)^{2}\right]\right\} \\
= & -2 \varepsilon \Delta \rho \omega^{2} R_{0} .
\end{aligned}
$$

The total pressure difference will then be

$$
\Delta p=\Delta p_{\text {Laplace }}+\Delta p_{\text {centr. }}=\frac{2 \gamma \varepsilon}{R_{0}^{2}}\left[1-X^{2}-A\right]
$$

where

$$
A=\frac{\Delta \rho \omega^{2} R_{0}^{3}}{\gamma} .
$$

From this equation it follows that a positive pressure difference and hence the break-up instability will only occur for $A<1$.

We can now apply again our simple model using Poiseuille's formula Eq. (8) to calculate the growth rate. This results in

$$
q=\frac{\gamma X^{2}\left(1-X^{2}-A\right)}{8 \pi \eta_{1} R_{0}} .
$$

The maximum growth rate and corresponding wavelength are now given by

$$
\begin{gathered}
q_{\mathrm{m}}=\frac{\gamma(1-A)^{2}}{32 \pi \eta_{1} R_{0}}, \\
X_{\mathrm{m}}=\sqrt{\frac{1-A}{2}} .
\end{gathered}
$$

These expressions show that with increasing rotation speed, in agreement with experiment [9], the rate of break-up decreases and the corresponding wavelength increases. For $A=1$ the growth rate becomes zero and the wavelength of the instability infinite. From the Vonnegut equation (2) however it follows that the liquid cylinder only becomes stable for a rotation speed such that $A=4$. Thus the liquid cylinder becomes 
stable against break-up in small droplets at rotation speeds that lie well below (to be precise a factor 2) the rotation speed that stabilizes them.

\section{References}

[1] E.H.A. de Hoog, H.N.W. Lekkerkerker, J. Phys. Chem. B 103 (1999) 5274.

[2] E.H.A. de Hoog, H.N.W. Lekkerkerker, J. Schulz, G.H. Findenegg, J. Phys. Chem. B 103 (1999) 10657.

[3] J.S. Rowlinson, B. Widom, Molecular Theory of Capillarity, Clarendon Press, Oxford, 1982.

[4] P.G. de Gennes, Scaling Concepts in Polymer Physics, Cornell University Press, Ithaca, NY, 1979.

[5] B. Vonnegut, Rev. Sci. Instrum. 13 (1942) 6.

[6] H.M. Princen, I.Y.Z. Zia, S.G. Mason, J. Colloid Interface Sci. 23 (1967) 99.

[7] Lord Rayleigh, Proc. London Math. Soc. 10 (1878) 4.

[8] S. Tomotika, Proc. R. Soc. Ser. A 150 (1935) 322.

[9] E.H.A. de Hoog, Thesis, Utrecht 2001 (Chapter 3). 